\title{
Mathematical Programming Approaches for Stable Tactical and Operational Planning in Supply Chain and APS Context
}

\author{
André Thomas* — Patrick Genin** — Samir Lamouri** \\ * Research Center for Automatic Control of Nancy, Henry Poincaré University \\ BP 239, Faculté des Sciences, F-54506 Vandouvre les Nancy Cedex \\ Andre.Thomas@cran.uhp-nancy.fr \\ ** Laboratoire d'ingénierie des structures mécaniques et des matériaux (EA2336) \\ ISM de Paris, 3 rue Fernand Hainaut, F-93407 Saint-Ouen Cedex \\ \{Patrick.Genin ; Samir.Lamouri\}@supmeca.fr
}

ABSTRACT. The economic context leads companies to answer quickly to the unpredictable changing customer demands. Advanced Planning Systems (APS) are useful tools to set up quickly Sales and Operations Planning (SOP) and Master Production Schedules (MPS). The problem is to obtain stable plans and schedules in spite of the unstable demand. In this paper, we propose a special procedure based on mathematical programming modelling to obtain stable MPS on the basis of a stable and robust SOP. The MPS process is built in two steps. The first leads to implement a SOP with a "reference plan policy". In the second step, SOP (quantity per family per month) is broken down into quantity of finished products per month, and then, into quantity of finished products per week (MPS). As we observe unstable MPS using Bitran and Hax procedure (1981), we propose a demand levelling stochastic treatment leading to better stability.

RESUMÉ. Le contexte économique conduit les entreprises à répondre rapidement aux demandes clients toujours plus imprédictibles. Les systèmes de planification avancée (APS) sont des outils utiles pour établir rapidement des plans industriels et commerciaux (PIC) et des programmes directeurs de production (PDP). Le problème est alors d'obtenir des plans et des programmes stables malgré une demande instable. Dans ce papier nous proposons une procédure particulière qui s'appuie sur de la modélisation mathématique pour obtenir des PDP stables et des PIC robustes. Le processus d'établissement des PDP est en deux étapes. La première conduit à implémenter un PIC par une "politique de plan de référence ». Dans la seconde, le PIC (quantité par famille par mois) est éclaté en quantité de produits finis par mois puis en quantité de produits finis par semaine (PDP). Nous avons obtenu des PDP instables en utilisant la procédure de Bitran et Hax (1981) ainsi nous proposons un traitement stochastique de lissage de la demande pour obtenir une meilleure stabilité.

KEYWORDS: Hierarchical Production Planning, Disaggregation, Linear optimisation, Nonlinear Optimisation, Robustness, Stability.

MOTS-CLÉS : planification hiérarchisée de la production, désagrégation, optimisation linéaire et non linéaire, robustesse, stabilité. 


\section{Introduction}

Most industrial companies try today to have a global view of their productiondistribution system. Supply Chain Management (SCM) aims to improve material, sites and market information utilisation in order to optimize material flows through the successive steps across the Supply Chain (SC) (Christopher, 1992). The centralisation of data through the use of ERP (Enterprise Resources Planning) highlighted the need for optimisation tools to manage the supply chain (APS Advanced Planning Systems) (Stadtler and Kilger, 2000). But this data coordination through the SC strengthens the need to tackle uncertainty properly.

At tactical level, Sales and Operation Planning (SOP) makes it possible to implement the strategic objectives defined by "Senior Management" in the Business Plan, by defining the production tasks that must be carried out in order to achieve the global objectives. SOP, established for each product family, leads to check whether the resources required are available on the factory floor. Thereafter, SOP must be disaggregated into several MPS (Master Production Schedules). A MPS is established for each finished product belonging to the SOP family to satisfy individual demand. Both SOP and MPS functionalities are named "Master Planning".

In industrial groups, a current problem is to distribute different volumes of products (or product types) that might be manufactured on different sites (Mukhopadhyay et al., 1998). All these calculations are carried out (often monthly) at the level of products family: SOP level (Vollman et al., 1997). SOP is entirely integrated into the commercial and information systems of demand management. It allows to put into practice the various master production schedules (De Kok and Inderfurth, 1997) and to take a large variety of constraints into account in order to produce optimized plans and schedules.

In general, managers use frozen periods and time fences to stabilise these plans and schedules. APS introduced the use of "dynamic horizons" (Stadtler and Kilger, 2000), which allow the frequent rescheduling of the various plans at all levels of the decision-making process throughout the supply chain. That leads to several changes in the SOP decisions, thus reducing the stability necessary for the plans at the operational level and throughout the supply chain.

Master Planning plays a key role in bullwhip effect, propagating demand information to suppliers through supply forecasts. Consequently, the goal at this level is to determine stable master schedules.

In a first research (Genin, 2003), we compared, at SOP level, different decision rules to achieve robust and stable supply chain plans considering that demand is exogenous. Alternatively, we suggested another way to achieve this objective. We proposed a special SOP policy named "the reference plan". The main idea in this approach was to introduce a plan, at the SOP level, which constrains plan changes so as to make it stable and robust. 
In a second research step (Ortiz, 2005), we proposed a SOP disaggregation procedure leading to obtain stable MPS coming from a stable SOP. This procedure using a two levels model takes into account stochastic demand variation and proposes a compromise between stocks and setup costs.

The objective of this paper is to describe this two steps procedure and to focus on the way to obtain these stable schedules. The proposed procedure is based upon mathematical programming modelling.

Before concluding this introduction, we would like to give a definition for "stability" and "robustness".

The term stability is generally opposed to "nervousness" (Blackburn et al., 1986; Ho, 1989; Minifie, 1990). De Kok and Inderfurth (De Kok and Inderfurth, 1997) have defined the term as "a lack of stability in the material requirements planning". Donselaar et al. (2000) compared the plans generated by MRP and by their heuristics in terms of nervousness. The considered indicator is the number of "replannings" encountered. The studied instability was the change within the periods. The instability of a plan is defined by the number of modifications made on the levels of decision parameters between two successive versions of the plan. The term stability is thus related to the number of changes between two successive generations of a plan.

The term robustness is generally associated with the ones of risk and decisionmaking (Kleijnen and Gaury, 2003). The underlying idea of system robustness is that the measured functions do not diverge significantly from a given value (Mulvey et al., 1995; $\mathrm{Yu}$ and $\mathrm{Li}, 2000)$.

According to (Lee and $\mathrm{Yu}, 1997)$, robustness is relative to one or several functions results and to their dispersion caused by uncertain parameters and costs. Robustness can be calculated by the standard deviation of each of the followed indicators.

Following this introduction, in Section 2 we review a bibliography concerning SOP robustness and stability, and Hierarchical Production Planning (HPP) processes. Section 3 describes the process allowing a stable production plan (stable SOP) using "reference plan policy". Consequently, as it is important to conserve the SOP stability quality after the disaggregation process is performed, a special procedure is described in this paper to obtain stable MPS (at the end of the Master Planning Process) on the basis of a stable SOP. The disaggregation process is built with two levels models. Successively, SOP (based on quantity per family per month) is broken down into quantity of finished products per month, and then, into quantity of finished products per week (i.e. MPS). Section 4 proposes this disaggregation process. To improve MPS stability, Section 5 proposes a stochastic treatment of the demand included in our disaggregation procedure. In the last section, we analyse the different results. 


\section{Bibliography}

\subsection{SOP robustness and stability}

APS offers functions spread over three levels of management: strategic, tactical and operational. Broadly speaking, the strategic level enables the definition of the total capacities of the production units in the company's Supply Chain (SC) namely: warehouses, providers, etc. (Shobrys and White, 2000). At the operational level, problems of production are addressed to control the activity in the short term.

The second level, tactical planning and especially SOP, leads to check whether the required resources (staff, stock, investments, etc.) are available on the factory floor. If this objective cannot be achieved, a decision will be taken to subcontract, for example. Managers traditionally use the following techniques to obtain stable plans.

\subsubsection{Planning time fences}

The principal approach for stabilizing plans consists in reducing the modifications. Stability is introduced by devices such as planning time fences and frozen horizons. A frozen horizon of $\mathrm{X}$ weeks means that no modifications are possible within these $\mathrm{X}$ weeks. In practice, the term "no modifications" is somehow strong. Companies have scant regard for the term "frozen". They consider that everything is negotiable. But negotiations become more difficult the nearer one gets to the present moment (Vollman et al., 1997).

The APICS dictionary defines a planning time fence as a policy or guideline established to note where various restrictions or changes in operating procedures take place (APICS, 1997).

The impact of time fences on the stability of plans is well known (Zhao and Lee, 1993), and we analyse in (Genin, 2003) the impact of this mode of management on the robustness of tactical planning.

\subsubsection{Management by exception supported by the APS}

Ho (1989) differentiates between the use of large and small modifications for stabilizing plans. The mode of management by exception leads to the management of exceptional events in the APS: an unexpected sale, significant changes in customer orders, the breakdown of a key machine, etc. A new plan is established, not at regular intervals, but in the event of consequent modifications. This procedure is often presented as being more effective (Stadtler and Kilger, 2000) and is thus used by companies having an APS.

This mode of management "dynamizes" the planning process by creating the necessary reactivity for any supply chain. However, just as for the planning horizons, the analysis of the impact of this mode of management on the robustness 
of the plans does not, to our knowledge, seem to have been studied. Genin showed also the benefits of such an approach (Genin, 2003).

\subsubsection{Sensitivity analysis}

APS creates plans by using a linear or a holistic modelling of tactical planning problems. The stability or the robustness of decisions can be determined by sensitivity analysis, resulting from linear programming. Koltai and Terlaky highlighted the differences in interpretations between the sensitivity of management and the mathematical analysis of sensitivity (Koltai and Terlaky, 2000).

The objective of such analysis is to study the effect of a change in the coefficients of the objective function and the effect of a change in the values of the constraints on the optimal value of the objective function, as well as the ranges of validity of these effects. The sensitivity analysis gives information on the interval of values of a parameter for which the solution remains optimal, and the rate of variation of the objective function. With regard to the problem of decision, the software should provide the information easily, which is seldom the case with APS when used to provide a sensitivity analysis.

Two criticisms can be made regarding sensitivity analysis: (1) this analysis, which is calculated in order to provide a solution, is carried out post-optimisation. It is not used as a criterion of optimization in the linear programming model and does not lead the model towards a robust solution; (2) the exact result of a linear programming model is sometimes not easily exploitable because its application requires a major re-evaluation of the existing situation. Next, it is interesting to compare the behaviour of the various optimal solutions. The software could be used to illustrate them, by showing the invariants and the alternatives in the decisions. To complete our criticism of the sensitivity analysis, it is significant to note that it works by acting on a parameter-by-parameter basis. It does not consider the effect of the interactions, and yet when several constraints exist, their simultaneous analysis is essential.

In this article, we address this problem and propose a method of tactical planning management, leading to more robust decision-making capacity within the framework of APS use. In this context, we have taken as our hypothesis the fact that APS applies linear programming as resolution method.

\subsection{Hierarchical Production Planning: context and problems}

The Hierarchical Production Planning (HPP) is a concept carried out in many research works. Hax and Candea (1984) introduced this concept in 1975. Their method primarily consists in recognizing the differences between tactical and operational decisions. The tactical decisions are associated with aggregate production planning while the operational decisions are an outcome of the 
disaggregation process. Hax and Candea proposed three levels of disaggregation: items, families and groups. Bitran et al. (1981) proposed the disaggregation process for an aggregated planning, considering the idea presented in (Hax and Candea, 1984). The model suggested by these authors uses the convex knapsack problems to disaggregate production. The first level consists of allocating production capacity among product types using an aggregate planning model. The planning horizon of this model normally covers a year in order to take in proper consideration the fluctuation demand requirements for the products. This model is solved with linear programming. At the second level, the disaggregation process takes into account the optimal production found in the first level to issue the production of families. In this first model, the objective is to minimize the total setup costs. Finally, the family production allocation is divided into the items belonging to each family (Figure 1). For the second model, an algorithm is proposed in (Bitran and Hax, 1981). (Erschler et al., 1986) and (Mercé, 1987) analysed these models on the basis of consistence, coherence and robustness criteria.

(Özdamar et al., 1996) propose the heuristic modification to (Bitran and Hax, 1977) algorithm and an alternative filling procedure to improve the infeasibilities in the disaggregation stage for the family production.

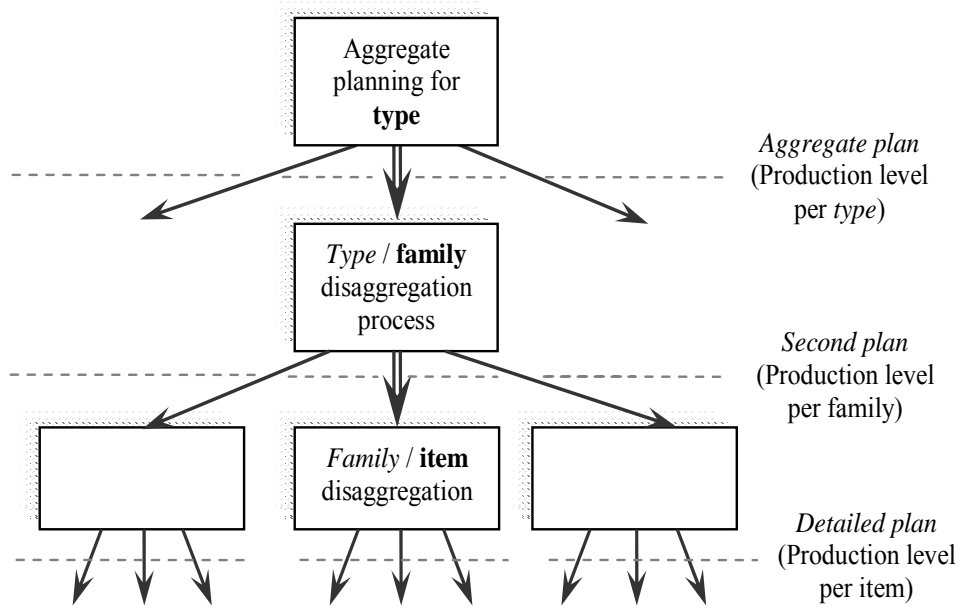

Figure 1. Bitran, Haas and Hax’s hierarchical structure (Bitran et al., 1981)

Giard (2003) examines the disaggregation problems in the context of the coherence for cases in which the products are not homogenous, in volume or structure in the family. Then, the principal problem is to coordinate the different levels to determine an optimal, robust and stable plan of disaggregation. 


\section{Proposition of useful master planning models}

Because of the changeable nature of master planning, the relevance of a decision made over a particular period of time can be judged only after several periods have elapsed. The simulation of an operating mode, that is flexible at the Master Planning level, thus appeared to be an interesting approach. In the following, we first propose a new approach named "reference plan" at the SOP level. Then, we propose to desegregate the obtained stable SOP plan into several stable MPS schedules with a methodology using a two levels model.

In (Genin, 2003), we proposed a special SOP procedure to obtain a stable and robust production plan. Figure 2 shows the general idea for this study. The aim of this procedure is to compare different indicators, such as service level, signal/noise ratio, inventory level, range or standard deviation, to characterize the objective function. We suggested that, with some variations in demand quantities, it is possible to obtain a stable and robust distribution of production planning quantities.

We defined robustness as the standard deviation of a function. If a policy 1 gives a standard deviation smaller than the one obtained by another policy 2, we will say that policy 1 is more robust that policy 2 . Stability has been defined as the number of changes in production quantities.

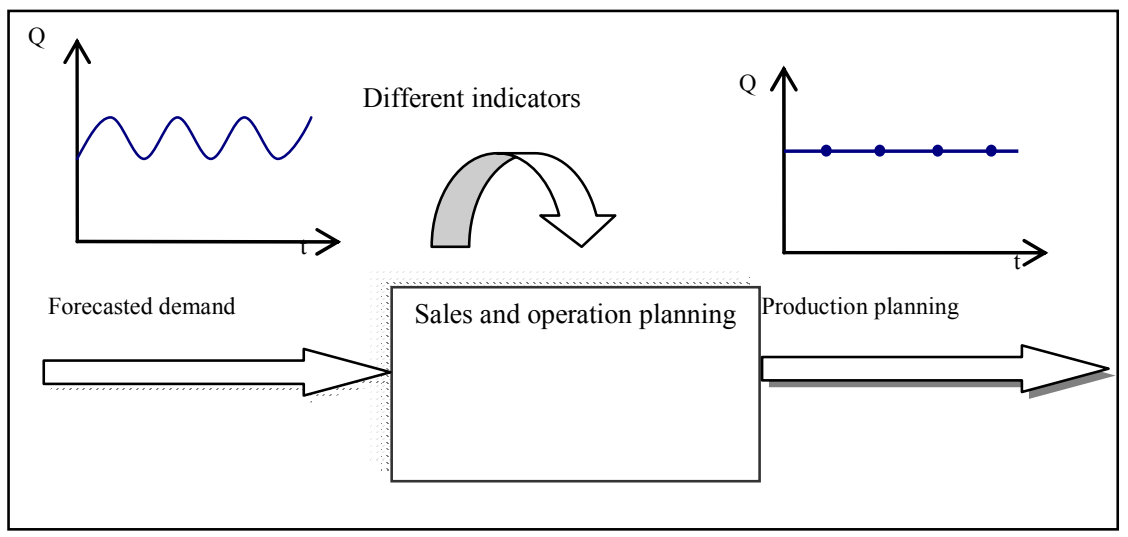

Figure 2. Concept of procedure for "reference plan"

Initially, in this first work, we carried out experiments for three SOP traditional management methods presented in Section 2.1. These experiments make it possible to highlight the strengths and weaknesses both of the existing practices (management with or without planning time fences) and of the emergent methods (management by exception implemented in APS). Here, our concern is to merely summarize the results. For a detailed development, we refer the reader to (Genin, 
2003). However, these simulations have enabled us to propose a new mode of operation: management by reference plan.

The presented SOP model is simplified; furthermore, this model of linear programming was used as a benchmark for our experiments.

\subsection{Notation}

\subsubsection{Indexes}

$T$ : tactical plan horizon,

$n$ : number of incremented periods during a replication,

$p$ : actual period of the simulation $p=1,2, \ldots, n$,

$t$ : month index of the plan $t=p, p+1, \ldots, p+T-1, t=p-1$ gives the initial state of the period $p$.

\subsubsection{Parameters defined in period $p$}

$d_{p}:$ actual family demand,

$F_{p t}$ : family forecast for the month $t$ known at the beginning of period $p$,

$u_{p t}^{P}$ : number of produced units by one worker during the month $t$,

$u_{p t}^{O}:$ number of produced units in one overtime hour for the month $t$,

$C_{p t}^{P}$ : maximum production capacity available for the month $t$, in unit,

$C_{p t}^{I}$ : maximum inventory capacity available for the month $t$, in unit,

$C_{p t}^{O}$ : number of overtime available per worker during the month $t$,

$i_{p T}$ : family inventory objective at the end of the horizon,

$b_{p T}$ : backorder objective at the end of the horizon,

$w_{p T}$ : worker number objective at the end of the horizon.

\subsubsection{Variables calculated in period $p$}

$I_{p t}$ : family inventory at the end of month $t$ (positive or null),

$B_{p t}$ : family backorders at the end of month $t$ (positive or null),

$R_{p t}$ : aggregated raw material inventory at the end of month $t$ (positive or null),

$W_{p t}$ : number of workers during the month $t$ (positive or null), 
$O_{p t}$ : overtime for the month $t$,

$H_{p t}$ : planed hiring for the month $t$ (positive or null),

$L_{p t}:$ planed dismissal for the month $t$ (positive or null),

$X_{p t}$ : number of family units produced during the month $t$ (positive or null),

$D_{p t}$ : delivered raw material units during the month $t$ (positive or null).

\subsubsection{Costs defined in period $p$}

$c_{p t}^{I}$ : family carrying cost for the month $t$,

$c_{p t}^{R}$ : aggregated raw material carrying cost for the month $t$,

$c_{p t}^{B}$ : family backorder cost for one produced unit for the month $t$,

$c_{p t}^{H}$ : hiring cost for the month $t$,

$c_{p t}^{L}:$ dismissal cost for the month $t$,

$c_{p t}^{W}$ : wages for one worker for the month $t$,

$c_{p t}^{O}$ : overtime cost for the month $t$,

$c_{p t}^{D}$ : aggregated raw material unit cost for the month $t$,

$c_{p t}^{T}$ : total cost of the month $t$.

\subsection{Basic model}

The model presented in this section relies on some simplifying hypothesis without lost of generality. It involves only one family of product and only one raw material considered as critical parts to be supplied. There is no explicit coefficient traducing how many units of raw material are used for producing one unit of the family. It has been considered as one with a supply lead time of one month (see [3]). The capacity is related to the available workforce according the numbers of working days and hours of overtime and the work performance.

\subsubsection{Cost function for a period $p$}

For each period $p$, the manager wants to minimize the total cost of the plan, $c_{p}^{T}$, subject to the constraints [2] to [10]. 


$$
\begin{gathered}
c_{p}^{T}=\sum_{t=p}^{p+T-1}\left(c_{p t}^{I} \times I_{p t}+c_{p t}^{B} \times B_{p t}+c_{p t}^{H} \times H_{p t}+c_{p t}^{L} \times L_{p t}\right. \\
\left.+c_{p t}^{W} \times W_{p t}+c_{p t}^{O} \times O_{p t}+c_{p t}^{R} \times R_{p t}+c_{p t}^{D} \times D_{p t}\right)
\end{gathered}
$$

The total cost is made of family inventory carrying cost, family backorder cost, hiring and dismissal costs, wages, overtime cost, aggregated raw material inventory cost, aggregated raw material purchasing and delivering cost.

\subsubsection{Constraints}

The modeling constraints are:

$$
\begin{aligned}
& I_{p, t-1}-B_{p, t-1}+X_{p t}=I_{p t}-B_{p t}+F_{p t} \quad \forall t, p \\
& R_{p, t-1}+D_{p t}-X_{p t}=R_{p t} \quad \forall t, p \\
& X_{p t} \leq I_{p, t-1} \quad \forall t, p \\
& W_{p, t-1}+H_{p t}-L_{p t}=W_{p t} \quad \forall t, p \\
& I_{p, p+T-1}=i_{p T} \quad B_{p, p+T-1}=b_{p T} \quad W_{p, p+T-1}=w_{p T} \forall p \\
& X_{p t} \leq C_{p t}^{P} \quad \forall t, p \\
& X_{p t} \leq u_{p t}^{P} \times W_{p t}+u_{p t}^{O} \times O_{p t} \quad \forall t, p \\
& I_{p t}+R_{p t} \leq C_{p t}^{I} \quad \forall t, p \\
& O_{p t} \leq C_{p t}^{O} \times W_{p t} \quad \forall t, p \\
& 0 \leq I_{p t}, B_{p t}, R_{p t}, W_{p t}, O O_{p t}, H_{p t}, L_{p t}, X_{p t}, D_{p t} \forall t, p
\end{aligned}
$$

[2], [2'] and [4] are the inventories and workers balance constraints. Expression [3] specifies that one can only use the raw material available at the beginning of a period. The stock, delay and worker objectives are given by [5], [6]. Expression [7] limits the labour and production capacity. Inventory constraint is [8]. Overtime is limited by [9]. All the variables are positive [10].

\subsection{Model with time fences}

In this model, a frozen zone is established, i.e. no modification of variables can be done. 
$f$ : the frozen zone, the number of months in which no change is implemented

$$
\begin{array}{ll}
W_{p t}=W_{p-1, t} & \forall p, \forall t \text { in [p..p+f-1] } \\
O_{p t}=O_{p-1, t} & \forall p, \forall t \text { in [p..p+f-1] } \\
X_{p t}=X_{p-1, t} & \forall p, \forall t \text { in [1..f] } \\
D_{p t}=D_{p-1, t} & \forall p, \forall t \text { in [1..f] }
\end{array}
$$

[11] to [14] constraint change in labour level, overtime, production and supply plan decisions. The objective function remains identical, subject to [2] to [14].

\subsection{Model with dynamic horizon}

In this case, an optimization of the whole plan is carried out only if an indicator reaches a predetermined threshold. In our model, the only existing uncertainty is the demand, leading to a variation in the levels of stock or delay. In practice, in order to be consistent with the used forecasting model, managers use the difference between the forecast and demand: the error (or bias) (Vollman et al., 1997). This indicator will trigger the alarm. The model previously described was modified to evaluate this indicator at more frequent intervals than at each period $p$. Each of the first four months of the planning horizon was divided into four sub-months, giving sixteen sub-months. The remaining periods of the planning horizon were left unchanged. Consequently, the forecasts $F_{p t}$ established for these months were linearly disaggregated over the four sub-periods. The capacities of the resources and storage, $C_{t}^{P}$ and $C_{t}^{I}$, the quantity of production per period, $u_{p t}^{P}$, the number of authorized overtime hours, $C_{p t}^{O}$ and the costs relating to backorders and storage, were also disaggregated linearly.

The indicator we choose to trigger a model optimization is the difference between the forecast of the sub-period and the actual demand of the current subperiod:

- If the absolute value of this difference is higher than two times the standard deviations, an optimization of the whole planning horizon is carried out (as in the basic case). This number " 2 " is arbitrarily selected. It has to result in the plan being reviewed only rarely because of the high level of dispersion of demand that can be reached before the alarm is triggered.

- If this condition is not achieved, the index of the period $p$ is incremented. In order to absorb the variation in the demand, an optimization is carried out over the first four sub-periods with the aim of reaching the level of inventory/backorder and number of operators for the fifth sub-month (the aim of the MPS is the achievement of its monthly objectives). If the sub-period is the first of a new period, optimization 
is carried out only over the eight previous periods (the SOP is then re-examined, taking a frozen horizon into consideration).

This procedure represents a situation in which events are absorbed by the decisions of operational management in order to avoid rescheduling the entire tactical plan. It respects the logic of hierarchical planning in which the lower level of planning seeks to achieve the volumes defined at the higher level.

Initially, we carried out experiments for the three traditional management methods. These highlighted the strengths and weaknesses both of the existing practices, (management with or without planning time fences) and of the emergent methods (management by exception). Here, our concern is to merely summarize our results. For a detailed development, we refer the reader to (Genin, 2003). However, these simulations led us to propose a new mode of operation which is the management by reference plan.

\subsection{Reference plan model: the role of stability in promoting robustness}

\subsubsection{Introduction of the concept}

The plan optimized in period $p$ is called $P_{p}^{O}$. This plan takes into account initial conditions (stock levels for example) and fixes the variables to minimize total costs. However, previous decisions taken and implemented could have perceptible effects only in later periods. For example, hiring some employees is decided in period $p-1$ for an impact in period $p+3$. This plan in progress, started previously, is called $P_{p}^{E}$. Differences can exist between those two plans according to events that have occurred. Traditionally, the manager modifies the plan $P_{p}^{O}$ according to plan $P_{p}^{E}$ in order to find an intermediate solution, which considers both previous and new conditions. Thus, this new plan $P_{p}^{R}$ is a compromise plan for the period $p$, so called "reference plan". It is plan which is implemented in period $p$ and serves as a framework to help the manager developing the tactical plan for the next period $p+1$ $\left(P_{p+1}^{E}=P_{p}^{R}\right)$. If the modifications are not significant, the reference plan is renewed for the following period. Otherwise, it is calculated as a compromise between the reference plan of the previous period and the optimized plan without reference to the preceding decisions.

\subsubsection{Modification of the basic model}

In this experiment, the modeling of the decision-making situation in basic model is modified to include the development of a reference plan, $P_{p}^{R}$ in the planning process. The manager's disgust to modify these decisions is represented by a weight on the modifications, i.e. on the difference between the new decision variables and 
those of the reference plan. The model seeks consequently a compromise between the cost induced by this difference and the cost of the new "ideal" plan.

The quantities to produce are chosen so that decision variables are stabilized. Actually, one of the main priorities of tactical planning is to stabilize production plan in order to limit the spread of variability. The production plan remains the reference plan as long as the control factors that influence it do not change significantly.

\subsubsection{Linear model with reference plan}

\subsubsection{Costs defined in period $\mathrm{p}$}

$c_{p t}^{P}:$ overcost for one extra family unit manufactured in relation with reference plan, for period $t$,

$c_{p t}^{N}$ : overcost for one family unit cancelled in relation with reference plan, for the period $t$.

The overheads evolution is decreasing (modifications for a near period are more expensive than a modification for the last periods of the plan).

\subsubsection{Variables calculated in period $p$}

$P_{p t}$ : extra unit manufactured in relation with reference plan, for period $t$,

$N_{p t}$ : unit cancelled in relation with reference plan, for period $t$.

\subsubsection{Cost function}

The manager's aim does not change, i.e. to minimize the overall cost. But now, the extra manufacturing and cancelling costs are included in the cost function. These overheads model the manager disgust to change his decisions.

The cost function becomes:

$$
\left.c_{p}^{T}=\sum_{t=p}^{p+T-1}+c_{p t}^{I} \times I_{p t}+c_{p t}^{B} \times B_{p t}+c_{p t}^{H} \times H_{p t}+c_{p t}^{L} \times L_{p t}+c_{p t}^{W} \times W_{p t}+c_{p t}^{D} \times D_{p t}+c_{p t}^{P} \times P_{p t}+c_{p t}^{N} \times N_{p t}\right) \quad \forall p
$$

In order to compare this policy with the other ones, cancelling or extra manufacturing costs are calculated like a result of the decision and not used to establish the plan in the other policies. They are added to the total costs.

\subsubsection{Additional constraints}

$$
\begin{aligned}
& D_{p t}=D_{p-1, t}-N_{p t}+P_{p t} \quad \forall p, t \\
& 0 \leq P_{p t}, N_{p t} \quad \forall p, t
\end{aligned}
$$


Expression [16] models the extra manufacturing or cancelling balance. All the variables are positive ([17]).

\subsection{Simulation}

\subsubsection{Values of the parameters}

Tactical plan calculated on a twelve months horizon: $h=12$. Frozen zone has been set to $3: f=3$.

The different data used for the simulations are given in Tables 1 and 2. They have been adapted from a real application in automotive industry (steel tubes).

Table 1. Tactical plan costs

\begin{tabular}{|l|c|c|c|c|c|c|}
\hline & 1 & 2 & 3 & $\ldots$ & 11 & 12 \\
\hline FG inventory cost & 20 & 20 & 20 & 20 & 20 & 20 \\
\hline Backorders cost & 60 & 60 & 60 & 60 & 60 & 60 \\
\hline Costs of hiring new employees & 400 & 400 & 400 & 400 & 400 & 400 \\
\hline Costs of layoffs & 500 & 500 & 500 & 500 & 500 & 500 \\
\hline Costs of operators & 1600 & 1600 & 1600 & 1600 & 1600 & 1600 \\
\hline Costs of overtime & 15 & 15 & 15 & 15 & 15 & 15 \\
\hline Procurement costs of raw materials & 200 & 200 & 200 & 200 & 200 & 200 \\
\hline Costs of stocks of raw materials & 10 & 10 & 10 & 10 & 10 & 10 \\
\hline Cancellation costs & 60 & 30 & 0 & 0 & 0 & 0 \\
\hline Express costs & 60 & 30 & 0 & 0 & 0 & 0 \\
\hline Forecast $F_{0 t}$ & 85 & 20 & 30 & 30 & 75 & 55 \\
\hline
\end{tabular}

For each case, 200 replications were launched in an identical way. The confidence interval at $95 \%$ on the costs accounts for $1 \%$ of the average value of the total costs. The parameters that are changed are the sets of forecasts and demands for the whole planning horizon. They were established once using a normal distribution for demand with a mean equal to the forecast and a standard deviation equal to a third of the forecasts. The forecasts were established using a single exponential smoothing with a coefficient 0.3 to create certain variability in demand. The forecasts shown in Table 1 are the initial set from which all the others have been computed. 
Table 2. Tactical plan data

\begin{tabular}{|l|c|}
\hline Opening hours/month/person & 160 \\
\hline Units/month/person & 8 \\
\hline $\begin{array}{l}\text { Maximum overtime } \\
\text { hours/month/worker }\end{array}$ & 20 \\
\hline Manufacturing time (hours/unit) & 20 \\
\hline Alpha & 0,3 \\
\hline $\begin{array}{l}\text { Initial stock of raw materials } \\
\text { (units), } t=0\end{array}$ & 0 \\
\hline
\end{tabular}

\begin{tabular}{|l|c|}
\hline Initial inventory (units), $t=0$ & 0 \\
\hline Inventory objective & 0 \\
\hline Initial number of employees, $t=0$ & 5 \\
\hline objective of number of employees & 5 \\
\hline machine capacity (units/month) & 200 \\
\hline Maximum stock (units) & 400 \\
\hline
\end{tabular}

In the case of simulation with a reference plan, the production plan for the period that is finishing is saved so that it can be used as input data in the model for the following period.

\subsubsection{Results analysis}

We note a reduction in costs, but also in the standard deviation, indicating a robustness improvement. The total costs of each replication are computed as the sum of the first month's costs for each planning iteration. The costs decrease by $3 \%$ and the standard deviation by $7 \%$ (Table 3 ). Reference plan policy leads to lower annual costs, while improving the robustness of raising the costs. There is a major difference in the service level indicator (Table 3). The policy with a reference plan results in an increase of more than $9 \%$ compared to the basic case. This result is obtained in this case by establishing a safety stock of raw material. Actually the model favours raw materials inventory build-up, even if it means incurring storage costs. This makes it possible to react more quickly to unforeseen demand without risking modification costs.

With regard to the indicator of stability (number of months the production quantities are changed), the advantage with the "reference plan" policy remains significant when compared to the basic case (Table 3). Thus, with this model, one gains a degree of freedom that is not possible using the frozen horizons which are traditionally used to reduce nervousness.

To summarize, we have included the costs bound to the modifications of the reference plan in order to allow comparisons to be made between the simulations of the traditional methods of management (with time fences and dynamic horizons).

Time fences policy leads to greater robustness in the plans and a reduction in the average costs. For these indicators, it is the most effective policy. The reference plan has an identical but less pronounced effect. Dynamic horizon and the reference policies have practically the same effect on the costs, causing them to increase because of the modifications made to the reference plans, and in this respect they are strategies to avoid. 
On the other hand, the indicator of robustness, the standard deviation, is different for each policy used. The dynamic horizon, the base case and the method of management with a reference plan lead to a certain robustness in planning. But frozen zones policy results in less robust plans because of its inertia to changes.

In our simulations, the inertia of the method of time fences policy leads to a strongly poor service level. Dynamic horizon and by reference plan policies make it possible to be regularly adjusted to demand, which enables them to ensure good service levels. It is interesting to observe that dynamic horizon policy leads to excellent service levels on some simulations. This is ensured by the iterations over the first sub-periods. The basic case is at an intermediate level. Table 3 presents a quantitative summary of the indicators for each mode of management.

The conclusions of this study could be influenced by the parameters used in the experiments. Although we did not realize an extensive experiment design, we made the same experiments with different sets of parameters. All of them give the same ranking of the policies. The differences are mainly quantitative. Depending of the costs values, the reference plan is more or less modified during the replications. Actually, that reflects the manager's sensibility to changes. The conclusions obtained for the traditional policies are on line with the results met in practice or checked by others researches.

Table 3. Performances of the studied policies for all of the simulations

\begin{tabular}{|c|c|c|c|c|}
\hline $\begin{array}{l}\ldots \quad \text { : best approach } \\
\ldots \quad \text { : second best } \\
\text { approach }\end{array}$ & $\begin{array}{l}\text { Basic } \\
\text { case }\end{array}$ & $\begin{array}{l}\text { Planning time fences } \\
\text { (Frozen horizon) }\end{array}$ & $\begin{array}{l}\text { Management by exception } \\
\text { (Dynamic horizon) }\end{array}$ & $\begin{array}{l}\text { Management by } \\
\text { reference plan }\end{array}$ \\
\hline Mean total costs & 245447 & 240120 & 249027 & 243894 \\
\hline Standard deviation & 33168 & 38504 & 28706 & $\underline{32900}$ \\
\hline Range of variation & $\underline{172667}$ & 190851 & 231511 & 171013 \\
\hline Mean service level & $73 \%$ & $51 \%$ & $78 \%$ & $\underline{71 \%}$ \\
\hline Stability & 3.3 & 0 & $\underline{1}$ & 1.6 \\
\hline
\end{tabular}

\subsubsection{SOP robustness conclusions}

The results are as expected for the first three policies: (1) planning time fences reduce reactivity (complete stability and low service levels) and lead to low costs when they include the costs of modifications; (2) the cost increases for management by exception, but so does robustness (reduction in the standard deviation); the same can be said for reactivity (increase in service levels), while preserving stability.

With regard to the chosen modelled policy, it seems that the method of management with planning time fences is the best adapted for minimizing the generation of variability upstream of the supply chain and thus for reducing the costs 
of the tactical plan. However, this policy is strongly disadvantaged by its low service levels and lack of robustness, with a large range of variation. The method of management by exception ensures a better service level while improving robustness, but to the detriment of costs (equivalent to the costs of the basic case) and stability. The reference plan policy therefore achieves a good compromise between these two approaches: low costs, excellent stability, good robustness and an intermediate service level. The reference plan also allows the range of cost variations to be considerably reduced, thus minimizing the risk taken by the manager.

In this first step, experiments were only made with a family according to the stability objective. Actually, we wanted to show that our "reference plan" approach leads to a stable production plan in spite of variation of the aggregated demand. In the second step, disaggregation process takes place considering the obtained stable production planning. The final objective is to show the effect of the proposed disaggregation procedure on the MPS stability.

\section{Disaggregation models}

We propose a two stages disaggregation procedure (see Figure 3). First, SOP is optimized to obtain the production and inventory quantities per family. In the first level, these production quantities are disaggregated into finished products for twelve months. The objective parameters of the model are: available inventory, overstock and safety stock. Effectively, the purpose of this level is to reach the inventories objectives established at the SOP level. The proposed model is considered as intermediate model. Then, the objective is to apply the disaggregation process to obtain finished product quantities to be analyzed into the second stage.

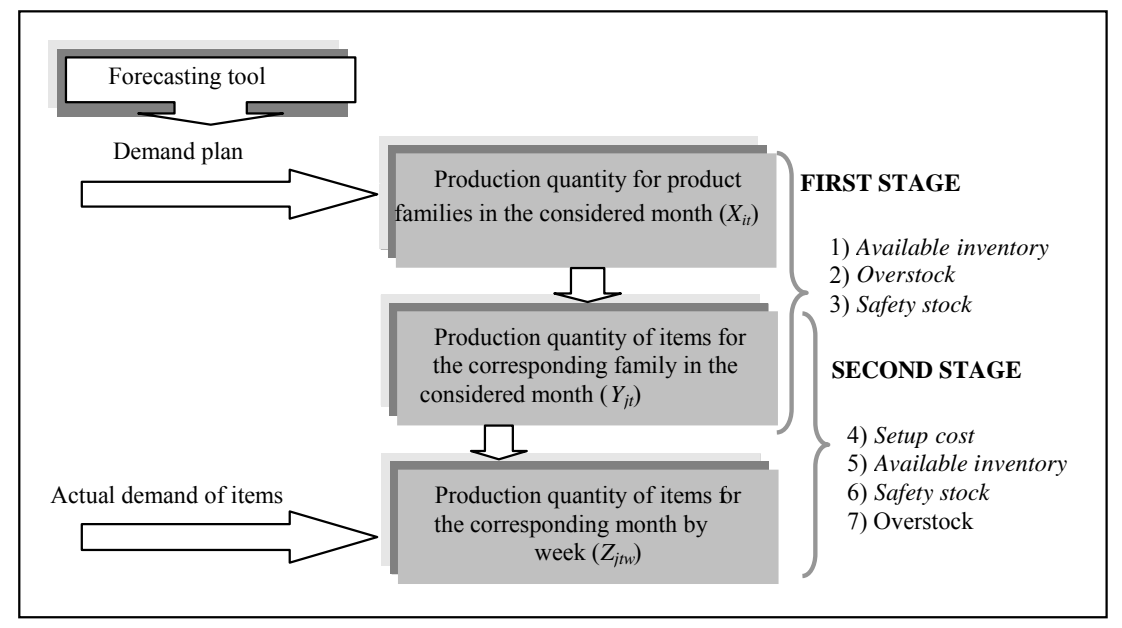

Figure 3. Disaggregation stages for SOP 
The second level considers finished product quantities found monthly. Corresponding safety stock, setup cost, available inventory and overstocks are considered. Actually setup costs minimization is strongly considered when releasing MPS propositions on the shop floor. The weekly MPS quantities are thus obtained at this level.

\subsection{Notation used in the disaggregation models}

$j$ : index for item,

$w$ : index for weeks,

$J(i)$ : set of items $j$ belonging to the product family $i$,

$W(t)$ : set of week $w$ for corresponding month $t$,

$i$ : index of product family, $i=1 . . I$,

$s s_{j t}$ : safety stock for item $j$ in month $t$,

$a i_{j t}$ : available inventory for item $j$ in month $t$,

$o s_{j t}:$ overstock limit for item $j$ in month $t$,

$d_{j t}$ : demand for item $j$ in month $t$,

$d_{j t w}$ : demand for item $j$ in week $w$ of month $t$,

$l b_{j t}$ : lower bound on production of item $j$ in month $t$ :

$l b_{j t}=\operatorname{Max}\left(0, d_{j t}-a i_{j t}+s s_{j t}\right)$,

$u b_{j t}$ : upper bound on production of item $j$ in month $t$, where $u b_{j t}=o s_{j t}-a i_{j t}$,

$s_{j t w}$ : setup cost for item $j$ in week $w$ of month $t$,

$X_{i t}^{*}$ : family quantity to be produced in month $t$ as optimized by SOP model,

$Y_{j t}^{*}$ : quantity of item $j$ to be produced in month $t$ as defined by the first stage model. In this case $i=1$ (only one family)

\subsection{Variables}

$Y_{j t}$ : production quantity of item $j$ in month $t$,

$Z_{j t w}$ : production quantity of item $j$ for month $t$ and week $w$.

\subsection{The family disaggregation model}

The model presented in this stage corresponds strictly to convex knapsack problem (Bitran and Hax, 1981). At this level, volumes (family products) obtained in SOP are disaggregated in production plans of items for the same period (month). It is thus important to minimize stock levels because of their impact on the income 
statement (and so, to obtain global cost reduction by economy of scale). The variables in this model are available and safety stock as well as overstock for the month. The central condition to be satisfied at this level is the equality between the sum of finished product and production quantities found in the SOP model [18]. This equality will assure consistency between the sales and operations planning and the family disaggregation process. Expression [19] guarantees lower and upper bounds to determine production quantities of items in the month.

Lower bound assures control of minimum volume to produce. This bound can take value zero if stock levels are high. This possibility does not lead to keep stability in determination of MPS. Upper bound allows verifying stock levels. On the other hand, this model is presented in a generic way and can be modified with respect to production policies. Expression [19] guarantees the limitation of finished product quantities to usable volume stock (for upper bound).

Besides, quadratic objective function allows keeping always the positive value. This function is composed of two terms. First term takes into account ratio between available quantities (volumes of family products and available inventories) and aggregated modified demand for the month. Second term uses the values of $Y_{j t}$ taking into account of available inventories and actual demand for the month. The calculation is realized for item of corresponding family. In others words, this second term gives a percentage of relative family quantities. If inventories are consumed, this model (with formulated constraints) will give, as optimal solution, demand values, i.e. production policy is lot-for-lot. Therefore, ratio for the two-terms taking value 1 , this function tends towards the minimal value of 0 .

$$
\operatorname{Min} \sum_{j \in J(i)}\left[\frac{X_{i t}^{*}+\sum_{j \in J(i)}\left(a i_{j t}-s s_{j t}\right)}{\sum_{j \in J(i)} d_{j t}}-\frac{Y_{j t}+a i_{j t}-s s_{j t}}{d_{j t}}\right]^{2}, t=1 \ldots T
$$

s.t.

$$
\begin{aligned}
& \sum_{J \in J(i)} Y_{j t}=X_{i t}^{*}, t=1 \ldots T \\
& l b_{j t} \leq Y_{j t} \leq u b_{j t}, j \in J(i), t=1 \ldots T \\
& l b_{j t}=\max \left(0, d_{j t}-a i_{j t}-s s_{j t}\right) \text { and } u b_{j t}=O s_{j t}-a i_{j t}
\end{aligned}
$$

\subsection{The item disaggregation}

The objective function [21] attempts to minimize the setup cost for each item per week taking into account of actual weekly demand. One significant hypothesis is that the monthly forecasted production quantity obtained at the SOP level is 
distributed into several weeks. Consequently several releases (and setups) are needed. This case occurs in all mass manufacturing contexts of customized products. At this level, objective function determines the number of releases, $d_{j t w} / Z_{j t w}$, in order to achieve production quantity of item for corresponding month and to minimize setup costs.

This solution is interesting if setup cost is high, if the company does not make MPS every week and moreover, if it does not seek to have stability in production volumes (for other formulations see (Ortiz and Thomas, 2004) and (Ortiz, 2005)).

The presented model is a non-linear problem. (Bitran and Hax, 1981) proposed an algorithm to solve this problem and (Özdamar et al., 1996) settled down some modifications for this procedure. We developed more this model and heuristic.

$$
\begin{aligned}
& \operatorname{Min} \sum_{w \in W(t)}\left(s_{j t w} * d_{j t w}\right) / Z_{j t w} \\
& \text { s.t. } \\
& \sum_{w \in W(t)} Z_{j t w}=Y_{j t}^{*} \\
& l b_{j t w} \leq Z_{j t w} \leq u b_{j t w}, w \in W(t), j \in J(i) ; t=1,2, \ldots, T
\end{aligned}
$$

The calculations lower and upper bounds are made by using the following conditions [24] and [25] proposed by (Erschler et al., 1986).

$$
\begin{aligned}
l b_{j t w} & =d_{j t w} \\
u b_{j t w} & =\sum_{t=1}^{T} d_{j t} \\
Z_{j t w} & =\left(\sqrt{s_{j t w} * d_{j t w}} / \sum_{w \in W(t)} \sqrt{s_{j t w} * d_{j t w}}\right) * Y_{j t}^{*}
\end{aligned}
$$

The function [21] is convex and differentiable, so the corresponding KuhnTucker conditions are applied to seek the expression [26]. Function [21] allows finding the values of $Z_{j t w}$ that are compared with the lower and upper bounds [23]. The procedure presented by (Özdamar et al., 1996) looks for avoiding the possible inconsistencies between the bounds and the values of finished product quantities in the corresponding week.

The application of this procedure is important to test the calculated values and to modify these quantities with the optimal conditions. 


\subsection{Application}

The proposed models are tested for one family and 15 items in a-year planning horizon. In this paper, only the results of the second model for three months are shown. CPLEX 10.0 is used to find the solutions to the first and second models.

According to the first step results (SOP), the production was levelled at 1200 units for each month of the production plan.

Table 4 contains the input data for the three corresponding months. The solutions for the intermediate level model are summarized in Table 5. At this level, it is possible to observe the disaggregation process of the family into the 15 items. As we said previously, the forecasted production quantities are stable, nevertheless, once the family is disaggregated, the behaviour of the distribution of finished products (items) quantities changes according to demand variation. The final objective is to check if the plan remains stable at the end of the disaggregation process.

Table 4. Input data for months 1 to 3

\begin{tabular}{|c|c|c|c|c|c|c|c|c|c|c|c|c|}
\hline \multirow[b]{2}{*}{$\stackrel{\mathscr{E}}{\Xi}$} & \multicolumn{4}{|c|}{ Month 1} & \multicolumn{4}{|c|}{ Month 2} & \multicolumn{4}{|c|}{ Month 3} \\
\hline & 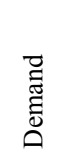 & 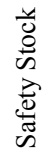 & 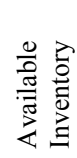 & 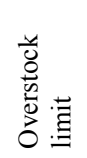 & 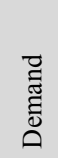 & 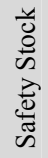 & 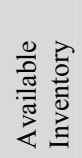 & 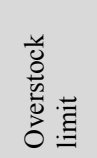 & 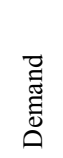 & 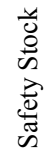 & 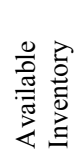 & 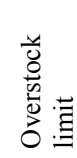 \\
\hline 1 & 85 & 25 & 80 & 5000 & 125 & 25 & 25 & 5000 & 125 & 25 & 25 & 5000 \\
\hline 2 & 60 & 50 & 40 & 5500 & 60 & 50 & 50 & 5500 & 60 & 50 & 50 & 5500 \\
\hline 3 & 80 & 40 & 0 & 8000 & 80 & 40 & 40 & 8000 & 65 & 40 & 40 & 8000 \\
\hline 4 & 85 & 50 & 50 & 7500 & 100 & 50 & 50 & 7500 & 50 & 50 & 50 & 7500 \\
\hline 5 & 70 & 60 & 20 & 9500 & 70 & 60 & 60 & 9500 & 150 & 60 & 60 & 9500 \\
\hline 6 & 80 & 45 & 60 & 6500 & 80 & 45 & 45 & 6500 & 80 & 45 & 45 & 6500 \\
\hline 7 & 70 & 25 & 60 & 7000 & 70 & 30 & 30 & 7000 & 80 & 30 & 30 & 7000 \\
\hline 8 & 65 & 50 & 35 & 9000 & 140 & 50 & 50 & 9000 & 140 & 50 & 50 & 9000 \\
\hline 9 & 80 & 40 & 60 & 9000 & 80 & 40 & 40 & 9000 & 90 & 40 & 40 & 9000 \\
\hline 10 & 100 & 50 & 15 & 10000 & 30 & 50 & 50 & 10000 & 30 & 50 & 50 & 10000 \\
\hline 11 & 85 & 60 & 10 & 10000 & 85 & 60 & 60 & 10000 & 60 & 60 & 60 & 10000 \\
\hline 12 & 90 & 45 & 10 & 8500 & 50 & 45 & 45 & 8500 & 50 & 45 & 45 & 8500 \\
\hline 13 & 75 & 25 & 50 & 8000 & 75 & 30 & 30 & 8000 & 25 & 30 & 30 & 8000 \\
\hline 14 & 50 & 50 & 60 & 9500 & 50 & 50 & 50 & 9500 & 50 & 50 & 50 & 9500 \\
\hline 15 & 60 & 40 & 40 & 6500 & 105 & 80 & 80 & 6500 & 50 & 80 & 80 & 6500 \\
\hline
\end{tabular}

For the third model, the input data are finished product values obtained in the second model (Table 5). These quantities are allocated to each week in the corresponding month. The setup cost varies between 20 and 40. In this paper, the analysis is realized on three items $(1,5$ and 11) for the considered family in 3 
months. The final results are shown in Figure 4. This figure shows the effects on the production plan once the disaggregation procedure is applied obtaining the master production scheduling. In the graph (a), production plan is stable (1200 units for each month) and the range for these production quantities is 0 . After carrying out the disaggregation procedure, stability existing in production plan is lost (see graph (b)). For this case, ranges for items 1,5 and 11 are 20,30 and 20 respectively. Consequently, this disaggregation procedure does not preserve stability.

Table 5. Results of family disaggregation for three months

\begin{tabular}{cccccccc}
\hline Items & Month 1 & Month 2 & Month 3 & Items & Month 1 & Month 2 & Month 3 \\
\hline 1 & 30 & 100 & 90 & 9 & 60 & 70 & 80 \\
2 & 70 & 70 & 70 & 10 & 135 & 20 & 40 \\
3 & 120 & 90 & 45 & 11 & 135 & 70 & 70 \\
4 & 85 & 130 & 80 & 12 & 125 & 35 & 55 \\
5 & 110 & 120 & 230 & 13 & 50 & 70 & 30 \\
6 & 65 & 45 & 75 & 14 & 40 & 40 & 60 \\
7 & 35 & 55 & 85 & 15 & 60 & 75 & 30 \\
8 & 80 & 210 & 160 & Total & 1200 & 1200 & 1200 \\
\hline
\end{tabular}

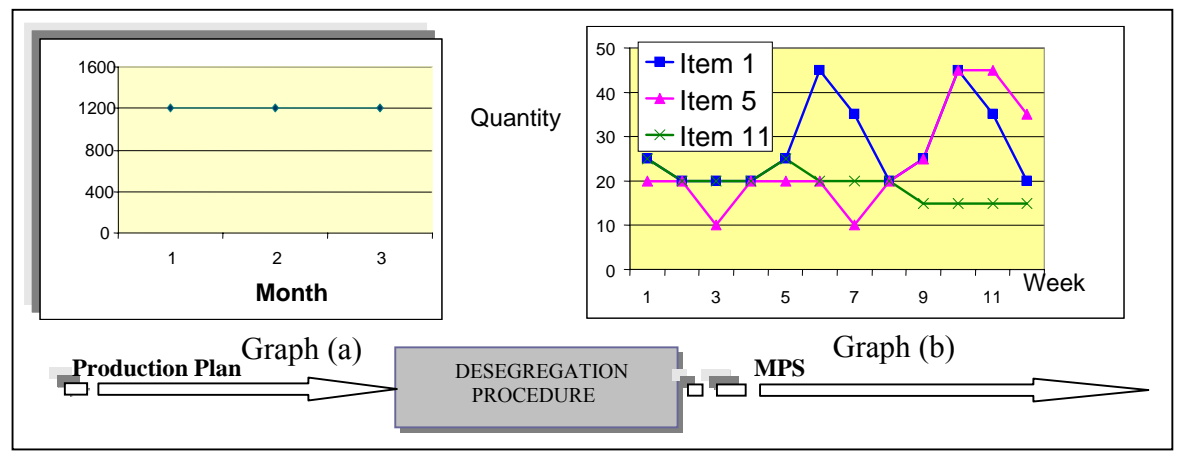

Figure 4. Comparison between production plan and MPS once applied disaggregation process 


\section{Model improvement}

As stability is not preserved, new disaggregation procedure is showed in Figure 5 .

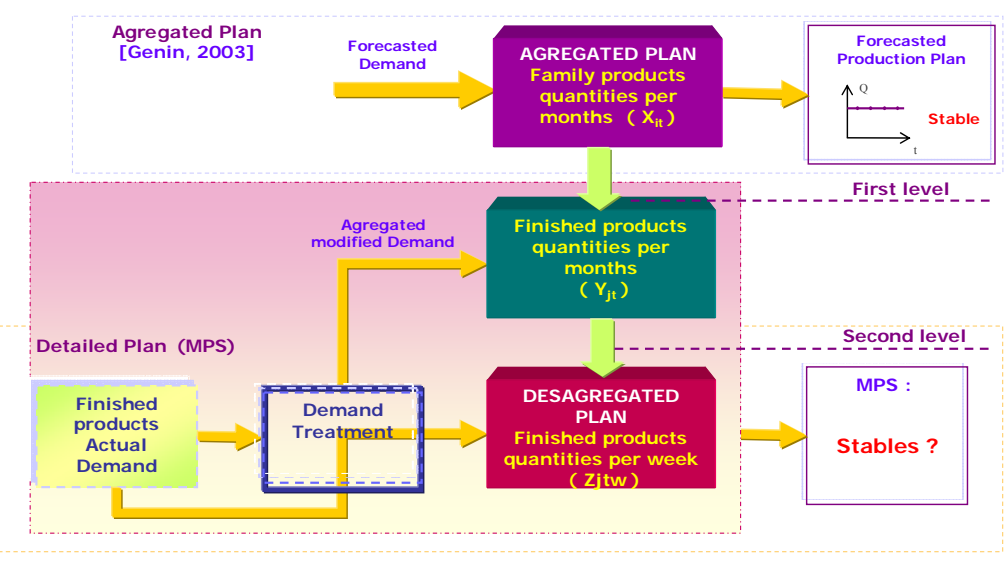

Figure 5. Disaggregation levels for the problem

In the second level, results obtained at the first level are introduced. Disaggregation process is used to determine weekly production quantity per item (for the monthly production quantity). The proposed methodology, at this level, consists of making a special treatment of actual demand of items to obtain stable MPS (we will describe this treatment in 5.4.1). This actual demand is aggregated and immediately introduced in the first level to determine new item production quantities for the corresponding month. It is important to observe that the disaggregation process is carried out with actual demand for each item in detailed plans.

\subsection{Notation used in the models}

\subsubsection{Parameters}

$d_{j t}^{A R}$ modified actual demand for item $j$ (AR demand - see 5.4.1) in month $t$ thanks to ARMA stochastic model,

$s s_{j w w}$ : safety stock for item $j$ in week $w$ of month $t$,

$o s_{j t w}$ : overstock limit for item $j$ in week $w$ of month $t$, 
$a i_{j t w_{0}}$ : initial available stock for item $j$ in month $t$, i.e. the first week $w_{0}$ of month $t$.

\subsubsection{Variables}

$Y_{j t}^{A R}$ : production quantity of item $j$ in month $t$ used AR(p) methodology,

$a i_{j t w}$ : available inventory for item $j$ in week $w$ of month $t$.

\subsection{First level: disaggregation of product family quantities in monthly items quantities}

The model presented in this stage corresponds strictly to convex knapsack problem described in Section 4.2 in which actual demand has been replaced by the modified (AR) demand.

$$
\operatorname{Min} \sum_{j \in J(i)}\left[\frac{X_{i t}^{*}+\sum_{j \in J(i)}\left(a i_{j t}-s S_{j t}\right)}{\sum_{j \in J(i)} d_{j t}^{A R}}-\frac{Y_{j t}^{A R}+a i_{j t}-s s_{j t}}{d_{j t}^{A R}}\right]^{2}
$$

s.t.

$$
\begin{aligned}
& \sum_{j \in J(i)} Y_{j t}^{A R} \geq X_{i t}^{*} \\
& l b_{j t} \leq Y_{j t}^{A R} \leq u b_{j t}, j \in J(i) ; t=1,2, \ldots, T \\
& l b_{j t}=\operatorname{Max}\left(0, d_{j t}^{A R}-a i_{j t}+s s_{j t}\right) \\
& u b_{j t}=\left(o S_{j t}-a i_{j t}\right)
\end{aligned}
$$

\subsection{Second level: determination of MPS (weekly items quantities)}

As in Section 4.3, the objective function [32] minimizes the setup cost for each item per week.

$$
\operatorname{Min} \sum_{w \in W(t)} \frac{S_{j t} \times Y_{j t}^{A R}}{Z_{j t w}}
$$

s.t. 


$$
\begin{aligned}
& \sum_{w \in W(t)} Z_{j t w}=d_{j t}-a i_{j t w_{0}} \\
& l b_{j t w} \leq Z_{j t w} \leq u b_{j t w}, w \in W(t), j \in J(i) ; t=1,2, \ldots, T \\
& l b_{j t w}=\operatorname{Max}\left(0, d_{j t w}-a i_{j t(w-1)}+s s_{j t(w-1)}\right) \\
& u b_{j t}=d_{j t w} \\
& Z_{j t w}>0, \forall j=1,2, \ldots, J ; t=1,2, \ldots, T ; w=1,2, \ldots, 4
\end{aligned}
$$

Input data are weekly actual demand, initial stock levels (available inventory and safety stock) and monthly volumes of items obtained at the first level.

Expression [33] establishes control between difference of monthly demand by item and the respective initial available inventory for the corresponding month. So this constraint allows satisfying demand in the month. Lower bound [35] provides, as in the model for the first level, maximum value to respect in manufacturing of items weekly considering used stock and demand for corresponding month. Upper bound limits the production according to the weekly demand [36]. In others cases, this constraint may be changed.

\subsection{Resolution}

The first level model is tested for one family with five items. The disaggregation procedure is realized for a-year planning horizon, with a two month frozen horizon. The solutions are obtained using CPLEX 10.0. To implement disaggregation procedure, we apply AR model to weekly item demand.

Second level model is a non-linear function. A heuristic was implemented to solve it, using the methodology explained in (Bitran and Hax, 1981) and (Özdamar et al., 1996).

\subsubsection{AR Model}

AR Methodology is based on (Box and Jenkins, 1976) proposition. It is a temporal series process, used to smooth series of values in the signal treatment community. As the disaggregation process doesn't maintain stability, AR methodology was used to modify the demand pattern (and consequently to increase or reduce production quantities) with the aim of smoothing the weekly item production quantities. Steps developed for our applications are: structure identification, parameters determination and model choice. These three steps are implemented using AR function of MATLAB 6.5.1. Data of actual weekly demand are input for AR function. This MATLAB function allows finding different polynomials depending of data, in our case eight at each time (because the frozen period is two-months, i.e. eight weeks). This methodology is repeated for every 
planning horizon. Finally, we choose polynomial model according to the minimum error. The obtained $\mathrm{AR}(\mathrm{p})$ prediction model for our data is:

$$
\hat{d}[k / k-1]=0,212 d[k-1]+0,313[k-2]+0,461 d[k-3]
$$

Where $\hat{d}[k / k-1]$ is demand prediction, degree of polynomial is three with FPE (Final Prediction Error) of 2083.64 and $\sigma_{\varepsilon}^{2}=13$.

The characteristic of $\operatorname{AR}(p)$ process is stationary. So, as polynomial root are interior unitary circle, stationary condition is proved and consequently, filter $\mathrm{H}(\mathrm{q})$ based over this polynomial is stable (for more details see (Ortiz, 2005)).

Figure 6 shows model behaviour for our demand data. The quantities found over demand for the corresponding week (week four) are stock levels and these quantities allow accomplishing every demand in the month. In the same manner, demand modified for weeks 6,7 and 8 is superior to the respective monthly demand. We show with this methodology that stability is obtained with generating stocks.

The model [38] is used to generate new weekly demands. The procedure to determine new values for the first model consists in incorporating new value of aggregated demand for the corresponding month (for our application, each month has four weeks). The sequence of steps carried out to obtain different MPS is showed in Figure 7.

\subsubsection{Proposition of a heuristic}

The second model is solved using the following proposed heuristic. As pointed out before, the objective function [32] proposed in second level is convex and differentiable, so the corresponding Kuhn-Tucker conditions are applied. We choose the approach suggested by (Özdamar et al., 1996). The details of the corresponding heuristic are also detailed in (Ortiz, 2005).

This heuristic consists in calculating possible values for $Z_{j t w}$, using the following expression:

$$
Z_{j t w}=\frac{Y_{j t}^{A R}}{4}
$$

This simple heuristic is used according to the fact that the production level smoothing is obtained through modifying the demand and not through optimizing the production quantity. This means that it could be possible to share evenly production volume of items (calculated in first level) over four weeks. 


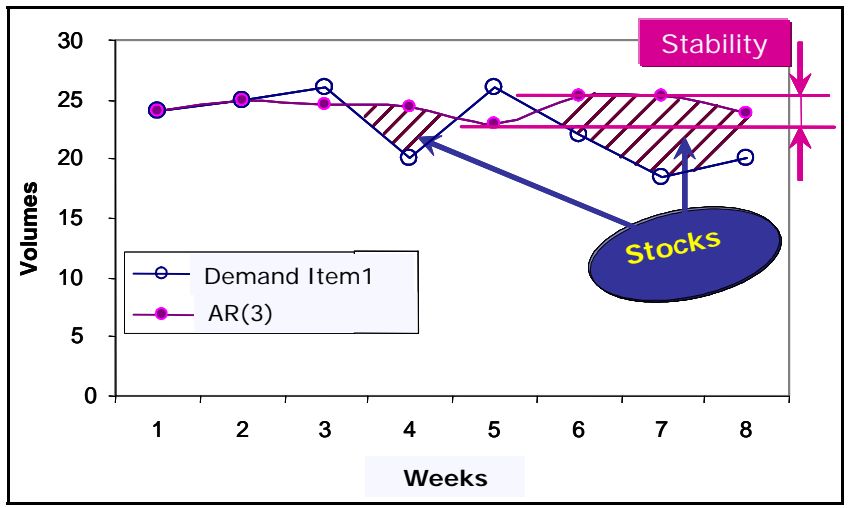

Figure 6. An example of modified demand for item 1

So, this condition [39] satisfies the "ideal hypothesis" of stability, useful for companies that do weekly MPS. Proposed heuristic allows finding optimal solutions respecting the constraints established in the model.

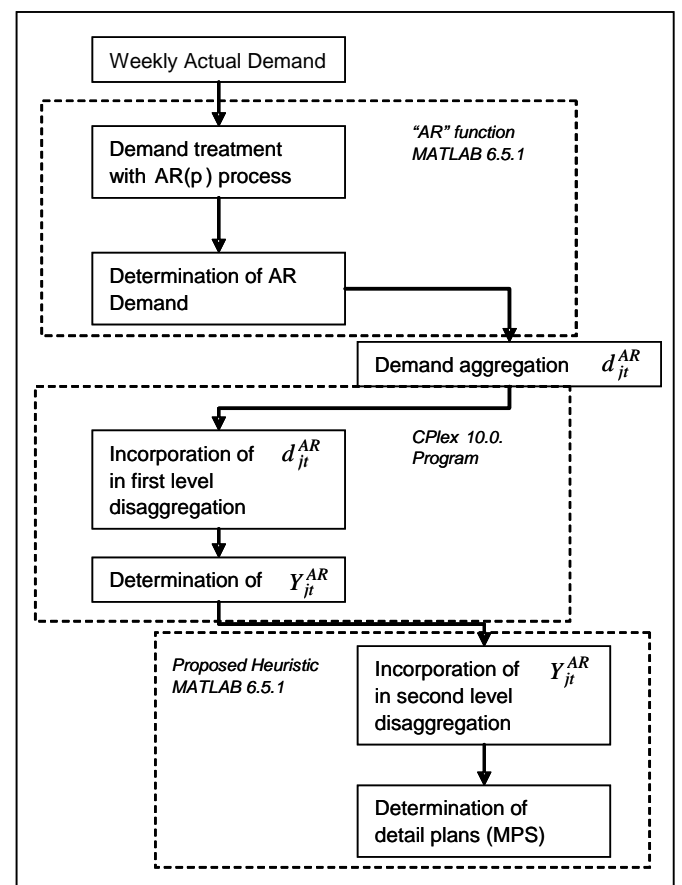

Figure 7. Stage sequence for applying disaggregation procedure 


\section{Results and discussion}

According to the first step results, the production was levelled at the following quantity: 1200 units for each month of production plan.

Figure 8 shows the behaviour of the detailed plan of item 1 for twenty eight weeks. It is possible to observe that available inventory levels are consumed. Item quantities found by disaggregation process are equal to actual demand. This situation may be seen since week fourteen. So, this production policy corresponds to "lot-for-lot policy".

Table 6 compares results obtained using a treatment model for real demand (method 2) and without treatment (method 1) for different items during eight weeks. We observe that item 1 has a better stability for different MPS with method 2. This result is corroborated with ratio value showed in Table 7 (ratio $=1.174)$. Item 5 does not present any significant improvement (ratio of method $1=1.063$ and method $2=1.083$ ). But, in a global analysis we also showed that items keeping more stability with the first method conserve it with the second method.

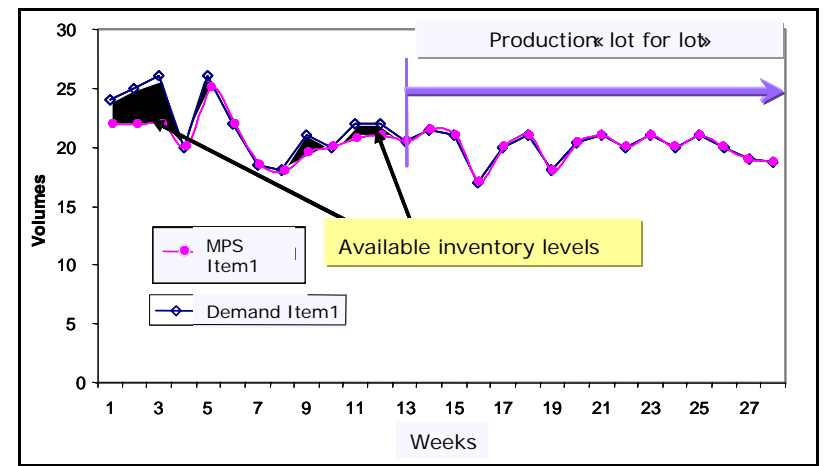

Figure 8. Behaviour of actual demand and detailed plan (MPS) for item 1 once applied disaggregation process

Table 6. Comparison between results obtained using method 1 and 2 for three items

\begin{tabular}{cccccccccc}
\hline Period & \multicolumn{3}{c}{ ITEM 1 } & \multicolumn{3}{c}{ ITEM 5 } & \multicolumn{3}{c}{$\begin{array}{c}\text { ITEM } \\
11\end{array}$} \\
\hline Weeks & $\begin{array}{c}\text { Actual } \\
\text { demand }\end{array}$ & $\begin{array}{c}\text { Method } \\
1\end{array}$ & $\begin{array}{c}\text { Method } \\
2\end{array}$ & $\begin{array}{c}\text { Actual } \\
\text { demand }\end{array}$ & $\begin{array}{c}\text { Method } \\
1\end{array}$ & $\begin{array}{c}\text { Method } \\
2\end{array}$ & $\begin{array}{c}\text { Actual } \\
\text { demand }\end{array}$ & $\begin{array}{c}\text { Method } \\
1\end{array}$ & $\begin{array}{c}\text { Method } \\
2\end{array}$ \\
\hline 1 & 24 & 21.3 & 22 & 25 & 18.3 & 19.8 & 24 & 20.6 & 22 \\
2 & 25 & 21.3 & 22 & 21 & 20.7 & 19.8 & 18 & 18 & 18 \\
3 & 26 & 22.4 & 22 & 16 & 16 & 16 & 30 & 27.4 & 26 \\
4 & 20 & 20 & 20 & 18 & 18 & 18 & 22 & 22 & 22 \\
5 & 26 & 26 & 25.1 & 17 & 17 & 17 & 22 & 22 & 22 \\
6 & 22 & 22 & 22 & 22 & 22 & 21.4 & 20 & 20 & 20 \\
7 & 18 & 18.5 & 18.5 & 17 & 17 & 17 & 21 & 21 & 21 \\
8 & 20 & 20 & 20 & 23 & 23 & 23 & 18 & 18 & 18 \\
\hline
\end{tabular}


Table 7. Comparison between actual demand and detailed plans ranges for some item

\begin{tabular}{cccccc}
\hline Type of item & $\begin{array}{c}\text { Range for } \\
\text { actual demand } \\
(\Delta)\end{array}$ & $\begin{array}{c}\text { Range detailed Range detailed } \\
\text { plan }(\delta) \\
\text { Method 1 }\end{array}$ & $\begin{array}{c}\text { Ratio }(\delta) \\
\text { Method 2 }\end{array}$ & $\begin{array}{c}\text { Rethod 1 } \\
\text { Meth }\end{array}$ & $\begin{array}{c}\text { Ratio }(\Delta / \delta) \\
\text { Method 2 }\end{array}$ \\
\hline Item 1 & 1.5 & 1.405 & 1.278 & 1.067 & 1.174 \\
Item 2 & 1.529 & 1.222 & 1.476 & 1.036 & 1.036 \\
Item 3 & 1.563 & 1.353 & 1.5 & 1.155 & 1.042 \\
Item 4 & 1.667 & 1.444 & 1.522 & 1.154 & 1.095 \\
Item 5 & 1.529 & 1.438 & 1.412 & 1.063 & 1.083 \\
\hline
\end{tabular}

\section{Conclusion}

The proposed methodology has two main objectives: first, to obtain a stable and robust Sales and Operations Plan; then, to disaggregate this stable production plan into different stable MPS. The disaggregation procedure formulated in this work improves the stability of detailed plan. This disaggregation procedure could be useful in an APS context.

The treatment model of the actual demand proposed in the disaggregation procedure helps to determine new weekly demands but lead to increasing stock levels. This methodology is an alternative solution to obtain more stable schedules. However the results are not convincing in all situations. Consequently, it will be interesting to specify other methods to obtain stable MPS. Is it possible to achieve this objective through an original disaggregation process? Is it possible without increasing average stock levels? Further researches on this subject are needed and we are working on different ways for smoothing item production quantities through an adaptive process taking into account demand variability, stocks levels and bottleneck capacity variability.

\section{Bibliographie}

APICS Dictionary, 1997

Bitran G.R., Hax A.C., "On design of hierarchical production planning systems", Decision Science, Vol. 8, 1977, p. 28-55.

Bitran G.R., Haas E.A., Hax A.C., "Hierarchical production planning: A single stage system", Operations Research, Vol. 29, No. 4, 1981, p. 717-743.

Bitran G.R., Hax A.C., "Disaggregation and resource allocation using convex knapsack problems with bounded variables", Management Science, Vol. 27, No. 4, 1981, p. 431441.

Blackburn J.D., Kropp H.D., Millen R.A., "Comparison of strategies to dampen nervousness in MRP systems”, Management Science, Vol. 32, No. 4, 1986, p. 413-429. 
Box G.E.P., Jenkins G.M., Time series analysis, forecasting and control, Holden-Day, Oakland, California, revised edition, 1976.

Christopher M., "Logistics and Supply Chain Management", Financial Times, Pitman, London, 1992.

De Kok A.G., Inderfurth K., "Nervousness in inventory management: comparison of basic control rules", European Journal of Operational Research, Vol. 103, 1997, p. 55-82.

Erschler J., Fontan G. and Mercé C., "Consistency of the disaggregation process in hierarchical planning”, Operations Research, 1986, Vol. 34, p. 464-469.

Donselaar K.V., Van Den Nieuvenhof J., Visschers J., "The impact of material coordination concepts on planning stability in supply chains", International Journal of Production Economics, Vol. 68, 2000, p. 164-176.

Genin P., Planification tactique robuste avec usage d'un APS, Thèse de Doctorat de l'Ecole des Mines de Paris, 2003.

Giard V., Gestion de la production et des flux, $3^{\mathrm{e}}$ éd., Economica, 2003.

Hax A.C., Candea D., Production and inventory management, Prentice-Hall, Inc. 1984.

Ho C., "Evaluating the impact of operating environments on MRP system nervousness", International Journal of Production Research, Vol. 27, 1989, p. 1115-1135.

Kleijnen J.P.C., Gaury E., "Short-term robustness of production management systems: A case study", European Journal of Operational Research, Vol. 148, 2003, p. 452-465.

Koltai T., Terlaky T., "The difference between the managerial and mathematical interpretation of sensitivity analysis results in linear programming", International Journal of Production Economics, Vol. 65, 2000, p. 257-274.

Lee J. H., Yu Z. H., "Worst-case formulations of model predictive control for systems with bounded parameters", Automatica, Vol. 33, 1997, p. 765-781.

Mercé C., Cohérence des décisions en planification hiérarchisée, Thèse de Doctorat d'Etat, Université Paul Sabatier, Toulouse, 1987.

Minifie J.R., Davis R.A., "Interaction effects on MRP nervousness", International Journal of Production Research, Vol. 28, 1990, p.173-183.

Mukhopadhyay S.K., Dwived Y.J. and Kumar A., "Design and implementation of an integrated Production Planning System for a pharmaceutical manufacturing concern in India", Production Planning and Control, Vol. 9, No. 4, 1998, p. 391-402.

Mulvey J.M., Vanderbei R.J.,. Zenios S.A, "Robust optimization of large-scale systems", Operations Research, Vol. 43, No. 2, 1995, p. 264-281.

Özdamar L., Atli A.Ö., Bozyel M.A., "Heuristic family disaggregation techniques for hierarchical production planning systems", International Journal of Production Research, Vol. 34, No. 9, 1996, p. 2613-2628.

Ortiz Araya V., Thomas A., "Proposition of disaggregation method for tactical robust planning", IFAC/IEEE Conference on Management and Control of Production and Logistics 2004, Santiago (Chili), November 2004. 
Ortiz Araya V., Proposition d'un modèle de désagrégation pour un plan tactique stable dans le contexte de Chaîne Logistique et de l'usage d'un APS, Thèse de Doctorat de l'Université Nancy I, 2005.

Stadtler H., Kilger C., Supply Chain Management and Advanced Planning: Concepts Models, Software and Case Studies, Stadtler H., Kilger C. (ed), Springer-Verlag, Berlin, 2000.

Shobrys D.E., White D.C., "Planning, scheduling and control systems: why can they not work together", Computers and Chemical Engineering Journal, Vol. 24, 2000, p. 163-173.

Vollman T.E., Berry W.L., Whybark D.C., Manufacturing planning and control systems, $4^{\text {th }}$ ed., New York et al., 1997.

Yu C.S., Li H.L., "A robust optimization model for stochastic logistic problems", International Journal of Production Economics, Vol. 64, 2000, p. 385-397.

Zhao X., Lee T.S., "Freezing the master production schedule for material requirements planning systems under demand uncertainty", Journal of Operations Management, Vol. 11, No. 2, 1993, p. 185-205. 